\title{
Hospitalization Risk According to Geriatric Assessment and Laboratory Parameters in Elderly Hematologic Cancer Patients
}

\author{
Kamile Silay ${ }^{1 *}$, Sema Akinci², Yavuz Selim Silay ${ }^{3}$, Tekin Guney ${ }^{2}$, Arife Ulas ${ }^{4}$, \\ Muhammed Bulent Akinci ${ }^{5}$, Esin Ozturk ${ }^{6}$, Merve Canbaz $^{6}$, Bulent Yalcin $^{5}$, Imdat \\ Dilek $^{5}$
}

\begin{abstract}
Background: Utilizing geriatric screening tools for the identification of vulnerable older patients with cancer is important. The aim of this study is to evaluate the hospitalization risk of elderly hematologic cancer patients based on geriatric assessment and laboratory parameters. Materials and Methods: In this cross sectional study 61 patients with hematologic malignancies, age 65 years and older, were assessed at a hematology outpatient clinic. Standard geriatric screening tests; activities of daily living (ADL), instrumental activities of daily living (IADL), Mini Nutritional Assessment (MNA), Mini Mental State Examination (MMSE), timed up and go test (TUG), geriatrics depression scale (GDS) were administered. Demographic and medical data were obtained from patient medical records. The number of hospitalizations in the following six months was then recorded to allow analysis of associations with geriatric assessment tools and laboratory parameters. Results: The median age of the patients, 37 being males, was 66 years. Positive TUG test and declined ADL was found as significant risk factors for hospitalization $(\mathrm{p}=\mathbf{0 . 0 2 8}$ and $\mathrm{p}=\mathbf{0 . 0 1 5}$ respectively). Correlations of hospitalization with thrombocytopenia, vitamin B12 and folic acid deficiency were statistically significant $(p=0.004, p=0.011$ and $p=0.05$ respectively). Conclusions: In this study, geriatric conditions which are usually unrecognized in a regular oncology office visit were identified. Our study indicates TUG and ADL might be use as predictive tests for hospitalization in elderly oncology populations. Also thrombocytopenia, and vitamin B12 and folic acid deficiencies are among the risk factors for hospitalization. The importance of vitamin B12 and folic acid vitamin replacement should not be underestimated in this population.
\end{abstract}

Keywords: Elderly - hematologic malignancy - geriatric assessment - hospitalization

Asian Pac J Cancer Prev, 16 (2), 783-786

\section{Introduction}

Approximately 60 percent of new cases and 70 percent of mortality from cancer occur in patient's $\geq 65$ years of age (Yancik et al., 2004; Castellon et al., 2006; Lichtman et al., 2006). Since the majority of elderly patients who have cancer were willing to receive chemotherapy, the care of older patients is an important part of everyday practice for the oncologist (Razali et al., 2013). Management of elderly patients with cancer requires a multidisciplinary approach. The routine use of a geriatric assessment for the older patient with cancer is recommended from both the National Comprehensive Cancer Network (NCCN) and the International Society for Geriatric Oncology (Extermann et al., 2005).

Geriatric conditions frequently unrecognized in older adults. Identifying geriatric conditions can help clinicians manage these conditions and prevent or delay their complications. Comprehensive geriatric assessment (CGA) is defined as a multidisciplinary diagnostic and treatment process that identifies medical, psychosocial, and functional limitations of a frail older person in order to develop a coordinated plan to maximize overall health with aging (Stuck et al., 1993; Devons et al., 2002). Since GA is time consuming many screening tools were developed, however; updated SIOG recommendations suggest screening tools in older cancer patients should not replace CGA (Decoster etal., 2014).

The typical domains of a CGA include evaluations of functional status, comorbid medical conditions, cognitive status, psychological state, social support, nutritional status, and a review of the medication list.

During the diagnosis and treatment planning clinicians may overlook the impact of the cancer and its treatment

${ }^{1}$ Department of Geriatrics, ${ }^{2}$ Department of Hematology, ${ }^{4}$ Department of Oncology, ${ }^{5}$ Internal Medicine, ${ }^{6}$ Department of Family Medicine, Ataturk Research and Training Hospital, Faculty of Medicine, Yildirim Beyazit University, ${ }^{3}$ Association of Research-Based Pharmaceutical Companies (AIFD), Nonprofit Organization, Ankara, Turkey*For correspondence: kamilesilay@hotmail.com 
on elderly cancer patient. Utilizing Geriatric Screening tools for the identification of vulnerable older patients with cancer is important. Providing adequate time and attention for systematic review of the physical, emotional, and social consequences of cancer can be an important first step in understanding the patient's response to the cancer experience. There is strong evidence that CGA improves function and reduces hospitalization in the elderly (Extermann et al., 2005). Also a CGA will be useful tool for medical decision making and adjusting treatment plan. It will help both clinician and patient to decide whether to continue treatment or consider dose reduction or palliative treatment.

The aim of this study is to evaluate the hospitalization risk of elderly hematologic cancer patients based on geriatric assessment and laboratory parameters.

\section{Materials and Methods}

This is a cross sectional study including 61 patients with hematologic malignancies, age 65 years and older who were assessed at hematology outpatient clinic. Standard geriatric screening tests were administered to assess mood, functional status, nutritional and cognitive status. The number of hospitalizations over 6 months was recorded. Demographic and medical data including hematologic and biochemical parameters were obtained from patient's medical records.

Functional status assessed by activities of daily living (ADLs) that represents the ability of the patient to take care of himself or herself, and instrumental activities of daily living (IADLs) that assess the ability of the patient to live independently in the community; cognitive status assessed using the Mini Mental State Examination (MMSE); nutritional status assessed by Mini Nutritional Assessment (MNA); psychological distress assessed by Geriatric Depression Scale (GDS), mobility and gait assessed by Timed Up and Go Test (TUG). The number of medications and comorbidities were recorded.

Hemoglobin levels $<12 \mathrm{~g} / \mathrm{dL}$ in women and $<13 \mathrm{~g} / \mathrm{dL}$ in men were considered as anemia, leukocytes $<4000 /$ microL as leukopenia, and platelets $<150 \times 109 / \mathrm{L}$ as thrombocytopenia. The reference ranges were $15-150 \mathrm{ng} /$ $\mathrm{mL}$ in women and $30-150 \mathrm{ng} / \mathrm{mL}$ in men for ferritin, 197$866 \mathrm{pg} / \mathrm{mL}$ for vitB12, and 4.6-18.7 ng/mL for folic acid.

Baseline demographics and geriatric assessment results were summarized using descriptive statistics. The relation between the number of hospitalizations and geriatric assessment tools and laboratory parameters were evaluated with chi square statistical analysis. For the statistics of the study SPSS.20 software was used.

\section{Results}

The median age of the patients was 69 years. There were 24 female and 37 male. Patients were divided according to their age in two groups to those between 65 and $74(n=48)$ and those 75 and older $(n=13)$.

About $21.3 \%$ and $26.2 \%$ had limitations on one or more ADL and IADL domains respectively. Geriatric problems including cognitive impairment (minimal
Table 1. Results of Geriatric Assessment Tests According to Age

\begin{tabular}{lrrr}
\hline & $\begin{array}{c}\text { Age 65-74 } \\
\mathrm{n}=48(78.7 \%)\end{array}$ & $\begin{array}{r}\text { Age }>75 \\
\mathrm{n}=13(21.3 \%)\end{array}$ & $\mathrm{p}$ \\
\hline Depression rate & $17(35.4 \%)$ & $4(30.8 \%)$ & 1.000 \\
Positive TUG & $6(12.5 \%)$ & $4(30.8 \%)$ & 0.198 \\
Impaired cognition & $9(18.8 \%)$ & $7(53.8 \%)$ & 0.028 \\
Limitations in ADL & $9(18.8 \%)$ & $4(30.8 \%)$ & 0.447 \\
Limitations in IADL & $11(22.9 \%)$ & $5(38.5 \%)$ & 0.297 \\
Polypharmacy rate & $23(47.9 \%)$ & $8(61.5 \%)$ & 0.534 \\
High comorbidity & $32(66.7 \%)$ & $12(92.3 \%)$ & 0.088 \\
Malnutrition risk & $12(25 \%)$ & $5(28.5 \%)$ & 0.486 \\
Hospitalization rate & $14(29.2 \%)$ & $2(15.4 \%)$ & 0.283 \\
\hline
\end{tabular}

cognitive impairment (4.5\%) and dementia (21.7\%)), risk of depression (34.4\%) and risk of malnutrition $(27.9 \%)$ were detected. The polypharmacy rate was found $50.8 \%$. The comorbidity score was found equals to 5 and more in $72.1 \%$ patients. The rate of positive TUG rate was $16.4 \%(\mathrm{n}=10)$.

The correlation between age and the result of geriatric assessment was analyzed (Table 1). Cognitive impairment was found associated with increased age $(p=0.028)$. The correlation of depression with comorbidity and ADL was found statistically significant $(\mathrm{p}=0.003, \mathrm{p}=0.006$ respectively).

The hospitalization rate was found $26.2 \%(n=16)$ over the six months period. The effect of age, ADL, IADL, comorbidity, TUG, MNA, polypharmacy, cognitive impairment and depression on hospitalization was evaluated with chi square analysis. Positive TUG test and declined ADL was found associated with hospitalization $(\mathrm{p}=0.028$ and $\mathrm{p}=0.015$ respectively).

The association between anemia, leukopenia, thrombocytopenia, low ferritin level, vitamin B12 and folic acid deficiencies, hyponatremia, hyperkalemia, hyper-hypocalcemia, and high creatinine level and hospitalization rate was also analyzed. The correlation of hospitalization with thrombocytopenia, vitamin B12 and folic acid deficiency was found statistically significant ( $\mathrm{p}=0.004, \mathrm{p}=0.011$ and $\mathrm{p}=0.05$ respectively). The anemia rate was $67.2 \%$ among the patients.

\section{Discussion}

In our study we evaluated geriatric domains that are not routinely assessed in oncology practice. We identified many geriatric conditions including dependencies in ADL and IADL, positive TUG, cognitive impairment and risk of depression which were unrecognized in a regular oncology office visit. Impairments in geriatric domains have predictive value for mortality and appear to be associated with toxicity (Hamaker et al., 2014). Our study enforces the evidence that geriatric assessment has utility regarding older adults with cancer.

The relation between these conditions and hospitalization rate were investigated in order to improve elderly cancer patient's care. The hospitalization rate of these patients over six months period was found $26.2 \%$. Positive TUG test and declined ADL was found as statistically significant risk factors for hospitalization 
Hospitalization Risk According to Geriatric Assessment and Laboratory Parameters in Elderly Hematologic Cancer Patients

( $\mathrm{p}=0.028$ and $\mathrm{p}=0.015$ respectively).

Our study indicates TUG and ADL might be use as a predictive test for hospitalization in elderly oncology population. Functional status refers to a patient's ability to perform routine daily tasks. The commonly used performance status scores (Karnofsky or Eastern Cooperative Oncology Group (ECOG) scales) tend to under-represent the degree of functional impairment in the older patient (Repetto et al., 2002). A more comprehensive understanding of an older patient's functional state can be obtained by ADL and IADL scales (Exterman et al., 2002; Khoei et al., 2013). In order to assess functional skills of older adults, TUG test is another well validated test. It is a simple one to administer and predicts ADL disability, falls, and decline in global health in older persons (Newmann et al., 2001). Also impaired functional status is associated with an increased risk of toxicity due to chemotherapy according to two multicenter studies (Hurria et al., 2011; Extermann et al., 2012). In both of these studies, the patient's functional status was a key predictor similar to our study.

Another important finding is the rate of depression. Approximately one-third of older patients with cancer experience psychological distress (Vinokur et al., 1990; Kua et al., 2005). This most typically takes the form of depression. The prevalence of clinically significant depression is estimated to be 3 to 25 percent in the older population with cancer (Kua et al., 2005). Depression rate was found $34.4 \%$ in our population. Since depression interferes with a person's ability to seek help, it might be easily overlooked in a routine visit if not specifically addressed. Addressing and treating depression will improve quality of life and prevent further functional and cognitive decline.

The relation between depression and comorbidity, ADL was found statistically significant ( $p=0.003, p=0.006$ respectively). Functional impairment increases in patients with late life depression, especially in those with multiple comorbidities (Katon et al., 2013). Our study revealed similar results in elderly cancer patients that depression is associated with poor performance status. The impact of comorbid medical conditions on life expectancy and treatment tolerance should be considered when estimating the risks and benefits of treatment (Welch et al., 1996). In our patient group comorbidity score was found high in $72.1 \%$ of patients.

Cognitive function is another important component of the overall assessment of the geriatric patient with cancer. There is growing evidence that cancer therapy may impact cognitive function, but few studies have focused on older adults (Hurria et al., 2011). In the general geriatric population, dementia is an independent prognostic factor for survival. In this study dementia rate was found $21.7 \%$. This information is very important since the presence of dementia influences the likelihood of both cancer diagnosis and treatment (Wolfson et al., 2001). Further and larger prospective studies are needed to evaluate the effect of chemotherapy on the cognitive status of elderly patients.

Nutritional status is an important prognostic factor in all geriatric patients. The MNA test is a simple, non invasive, well-validated screening tool for malnutrition in the elderly and is recommended for early detection of risks of malnutrition (Allepaerts et al., 2014). In the general older population, there are several reports demonstrating the adverse impact of weight loss or low body mass index (BMI) even when subjects do not have cancer (Wallace et al., 1995; Reynolds et al., 1999; Landi et al., 2000; Grabowski et al., 2001; Newman et al., 2001). The risk of malnutrition was found $27.9 \%$ in our population but no correlation was found between malnutrition risk and hospitalization.

A careful review of medications is an important component of the CGA. Although medications are an important factor in improving and maintaining the quality of life of older adults, polypharmacy increases the risks of morbidity and mortality, loss of functional independence, and a multiplicity of cognitive and physical problems (Chiang-Hanisko et al., 2014). A regular review of the medication list is needed to remove any unnecessary medications and to assess for potential drug interactions (Vestal et al., 1997; Lichtman et al., 2000). Half of the patients who were included in our study were taking more than 4 medications at the time of evaluation. One study showed that polypharmacy was not associated with chemotherapy-related toxicity or hospitalization in older adults with cancer (Maggiore et al., 2014). Our study also supports these findings since no correlation was found between polypharmacy and hospitalization.

The correlation of hospitalization with thrombocytopenia, vitamin B12 and folic acid deficiency was found statistically significant $(\mathrm{p}=0.004, \mathrm{p}=0.011$ and $\mathrm{p}=0.05$ respectively). The importance of vitamin B12 and folic acid vitamin replacement should not be underestimated in this population.

This study revealed that geriatric assessment for hematologic cancer patients is a useful tool for identifying geriatric conditions as well as the patients who are at risk for hospitalization. Our study indicates GA is not only useful prior to cancer treatment but also during the management period. This important piece of information could be useful to decide whether to continue treatment or consider dose reduction or palliative treatment. This would also help clinicians to decrease hospitalization rate with early intervention by recognizing risk factors. Our limitation is heterogeneous patient population since different types of hematological cancers were included.

In conclusion; geriatric assessment tools especially ADL and TUG will be useful for identification of older patients who are at risk for hospitalization. We recommend using ADL and TUG as predictive tests for hospitalization. Routine geriatric assessment may impact outcomes and often influences treatment decision of older patients with cancer. We suggest repeated CGA for every oncology patient age 65 and older. General geriatric data identify repeated geriatric assessments as a key component of effective geriatric interventions. We would like to emphasize the importance of replacing vitamin deficiencies such as vitamin B12 and folic acid in this specific patient population. Future research should study the predictive validity of these parameters by following patients prospectively. 


\section{References}

Allepaerts S, De Flines J, Paquot N (2014). Nutrition in the elderly. Rev Med Liege, 69, 244-50.

Castellon TD, Sieving KE (2006). An experimental test of matrix permeability and corridor use by an endemic understory bird. Conserv Biol, 20, 135-45.

Chiang-Hanisko L, Tan JY, Chiang LC (2014). Polypharmacy issues in older adults. $\mathrm{Hu} \mathrm{Li} \mathrm{Za} \mathrm{Zhi,} \mathrm{61,} \mathrm{97-104.}$

Decoster K, Van Puyvelde S, Mohile U, et al (2014). Extermann Screening tools for multidimensional health problems warranting a geriatric assessment in older cancer patients: an update on SIOG recommendations; An Oncol Advance, Access published June 16,

Devons CA (2002). Comprehensive geriatric assessment: making the most of the aging years. Curr Opin Clin Nutr Metab Care, 5, 19-24.

Extermann M, Hurria A (2007). Comprehensive geriatric assessment for older patients with cancer. J Clin Oncol, 25, 1824-31.

Extermann M, Aapro M, Bernabei R, et al (2005). Use of comprehensive geriatric assessment in older cancer patients: recommendations from the task force on CGA of the international society of geriatric oncology (SIOG). Crit Rev Oncol Hematol, 55, 241-52.

Extermann M, Boler I, Reich RR, et al (2012). Predicting the risk of chemotherapy toxicity in older patients: the chemotherapy risk assessment scale for high-age patients (CRASH) score. Cancer, 118, 3377-86.

Grabowski DC, Ellis JE (2001). High body mass index does not predict mortality in older people: analysis of the longitudinal study of aging. J Am Geriatr Soc, 49, 968.

Hamaker ME, Prins MC, Stauder R (2014). The relevance of a geriatric assessment for elderly patients with a haematological malignancy-a systematic review. Leuk Res, 38, 275-83.

Hurria A, Togawa K, Mohile SG, et al (2011). Predicting chemotherapy toxicity in older adults with cancer: a prospective multicenter study. J Clin Oncol, 29, 3457-65.

Katon WJ (2003). Clinical and health services relationships between major depression, depressive symptoms, and general medical illness. Biol Psychiatry, 54, 216-26.

Khoei MA, Akbari ME, Sharifi F, Fakhrzadeh H, Larijani B (2013). Translation and validation of the activities of daily living scale with Iranian elderly cancer patients treated in an oncology unit. Asian Pac J Cancer Prev, 14, 2731-7.

Kua J (2005). The prevalence of psychological and psychiatric sequelae of cancer in the elderly-how much do we know? Ann Acad Med Singapore, 34, 250-6.

Landi F, Onder G, Gambassi G, et al (2000). Body mass index and mortality among hospitalized patients. Arch Intern Med, 160, 2641-4.

Lichtman SM, Villani G (2000). Chemotherapy in the elderly: pharmacologic considerations. Cancer Control, 7, 548.

Lichtman SM (2006). Therapy insight: therapeutic challenges in the treatment of elderly cancer patients. Nat Clin Pract Oncol, 3, 86-93.

Maggiore RJ, Dale W, Gross CP, et al (2014). Polypharmacy and potentially inappropriate medication use in older adults with cancer undergoing chemotherapy: effect on chemotherapyrelated toxicity and hospitalization during treatment. $J \mathrm{Am}$ Geriatr Soc, 62, 1505-12.

Newman AB, Yanez D, Harris T, et al (2001). Weight change in old age and its association with mortality. J Am Geriatr Soc, 49, 1309-18.

Razali RM, Bee PC, Gan GG (2013). Survey of willingness to accept chemotherapy among elderly Malaysian patients.
Asian Pac J Cancer Prev, 14, 2029-32.

Repetto L, Fratino L, Audisio RA, et al (2002). Comprehensive geriatric assessment adds information to Eastern cooperative oncology group performance status in elderly cancer patients: an Italian group for geriatric oncology study. J Clin Oncol, 20, 494-502.

Reynolds MW, Fredman L, Langenberg P, Magaziner J (1999). Weight, weight change, mortality in a random sample of older community-dwelling women. $J$ Am Geriatr Soc, 47, 1409-14.

Stuck AE, Siu AL, Wieland GD, et al (1993). Comprehensive geriatric assessment: a meta-analysis of controlled trials. Lancet, 342, 1032-6.

Vestal RE (1997). Aging and pharmacology. Cancer, 80, 130210.

Vinokur AD, Threatt BA, Vinokur-Kaplan D, Satariano WA (1990). The process of recovery from breast cancer for younger and older patients. changes during the first year. Cancer, 65, 1242-54.

Wallace JI, Schwartz RS, LaCroix AZ, et al (1995). Involuntary weight loss in older outpatients: incidence and clinical significance. J Am Geriatr Soc, 43, 329-37.

Welch HG, Albertsen PC, Nease RF, et al (1996). Estimating treatment benefits for the elderly: the effect of competing risks. Ann Intern Med, 124, 577-84.

Wolfson C, Wolfson DB, Asgharian M, et al (2001). A reevaluation of the duration of survival after the onset of dementia. N Engl J Med, 344, 1111-6.

Yancik R, Ries LA (2004). Cancer in older persons: an international issue in an aging world. Semin Oncol, 31, 128-36. 